\section{Effect of intra-articular hyaluronic acid agents on subsequent rate of infection following total knee arthroplasty}

\author{
Robert J. Petrella,' Dhiren Mahadeva ${ }^{2}$ \\ 'Faculty of Medicine and Dentistry, \\ Lawson Research Institute, University of \\ Western Ontario, London, Ontario, \\ Canada; '2Department of Orthopaedics, \\ New Cross Hospital, Wolverhampton, \\ England, UK
}

\begin{abstract}
The aim of our study was to discover any relation between infection, including deep infections, after knee total joint arthroplasty in tive infection (bacterial) following total knee arthroplasty with previous exposure to intraarticular HA derivative agents. The aim of this study was to discover any relationship between the two.
\end{abstract} patients who had previous intra-articular hyaluronic acid injections compared to those who had not. We performed a retrospective review of 1776 patients who had osteoarthritis and received hyaluronic acid injections and then a subsample of 415 patients who subsequently underwent total joint arthroplasty: a large primary researchable database from 2002-2008. Surgery was conducted in a university academic network, while the hyaluronic acid injections were delivered in a large primary care arthritis referral center. The 415 patients had at least one year follow-up after total joint arthroplasty. Outcomes included demographics, pre-post total joint arthroplasty Western Ontario McMaster score, and clinical outcomes (knee flexion and extension), as well as adverse events including infection rates. Infections were determined on clinical grounds and confirmed with laboratory investigations. In the injection group there were 18 cases of infected total joint arthroplasty versus 21 in the no-hyaluronic-acid-injection group. However, when the type of hyaluronic acid derivative was considered, more infections with higher versus lower molecular weight (15 vs. 3) were observed. Hence, prior intra-articular hyaluronic acid does not increase the risk of subsequent infection post total joint arthroplasty.

\section{Introduction}

Intra-articular injections of hyaluronic acid (HA) derivative agents are utilized currently in the treatment of mild-to-moderate osteoarthritis of the $\mathrm{knee}^{1-4}$ and other degenerative joints. ${ }^{5-8}$ While there have been reports on longer-term efficacy, ${ }^{9-10}$ often HA is used as a temporary measure to delay more definitive surgery while producing adequate symptom control. As with any other treatment modalities, complications including infection are associated with its use. ${ }^{11-13}$ This is of particular concern because thorough aseptic technique is variable ${ }^{14}$ in settings delivering intra-articular HA. While the HA derivative agent is sterilized and delivered under aseptic conditions, often it is administered in an out-patient setting under variable sterile conditions. Most HA derivatives are administered over three to five consecutive weeks, thereby potentially augmenting the potential risk. ${ }^{3-5}$ Current estimates for infection post total joint arthroplasty (TJA) are between $2.9 \%$ (superficial) and $0.8 \%$ (deep).${ }^{15}$ However, there are no published studies that have correlated the rate of postopera-

\section{Materials and Methods}

We performed a retrospective review of the patients in a large primary care researchable database $^{16}$ who had received a TJA between 2002 and 2008. We identified those patients who had (HA) or had not received intra-articular HA (no HA) in the two years prior to surgery. All patients identified were compared for demographic characteristics, comorbidity using the Charlson index ${ }^{17}$ knee joint physical examination (flexion/extension), and Western Ontario McMaster score (WOMAC) ${ }^{18}$ (pre- and one year post-surgery). We excluded patients who had previous operations on the same knee, including high tibial osteotomy, patellectomy, and cruciate ligament reconstruction, and patients who had malignancy, rheumatoid arthritis, osteonecrosis, or previous intraarticular steroid infiltration. In addition, patients who had documentation of infections in the knee or even suspected infection prior to surgery were excluded. All patients in both HA and no-HA groups were at least a year postsurgery, which included a one-year postoperative follow-up assessment.

All patients for knee arthroplasty were selected critically and had shown that they exhausted conservative therapy. In the preoperative assessment, any focus of sepsis was screened for routinely. The knee replacement surgery was carried out in a laminar flow theater. Patients had a loading dose of antibiotic and further postoperative doses as per protocol. A standard midline incision, followed by a medial parapatellar approach was used to gain access to the joint. All implants were inserted with the use of a tourniquet. Antibiotic AKZ
Correspondence: Robert J. Petrella, 801 Commissioners Road East, Suite 3002, London, Ontario N6A 5J1, Canada. E-mail: petrella@uwo.ca

Key words: knee arthroplasty, hyaluronic acid.

Received for publication: 7 July 2009.

Revision received: 2 October 2009.

Accepted for publication: 13 October 2009.

This work is licensed under a Creative Commons Attribution 3.0 License (by-nc 3.0).

(C) Copyright R.J. Petrella and D. Mahadeva, 2009 Licensee PAGEPress, Italy

Rheumatology Reports 2009; 1:e8 doi:10.4081/rr.2009.e8

simplex P (Stryker, East Rutherford, NJ) bone cement was used. An intramedullary rod in the femoral cuts, extramedullary guide in the tibial cuts, and a spacer block were made to assume appropriate ligament balance. All patients underwent a standardized rehabilitation program, which consisted of early range of motion and weight-bearing exercises as tolerated. All patients also received prophylactic anticoagulation medication and antibiotics as necessary.

Intra-articular HA was administered as previously described. ${ }^{19}$ Briefly, under aseptic conditions and the knee flexed to 90 degrees, a 25 23 gauge $1 \frac{1}{2}$ in needle was inserted into the intra-articular space using a medial approach. Aspiration was conducted at the time to ensure adequate positioning of the needle within the intra-articular space. The contents of the needle were administered using a steady flow, and patients were counseled regarding signs of infection post-administration. Analgesics were utilized as needed. The presence of infection was based on clinical symptoms and signs corroborated with appropriate laboratory indices.

Analysis of variance with repeated measures and the $\chi^{2}$-test were used to test for differences between groups at baseline and postTJA. Analyses were conducted using Sigma Stat (SPSS, Chicago, IL, USA) and Microsoft Excel (Microsoft, Redmond, WA, USA). Changes in WOMAC-VAS were calculated in percentages of improvement from baseline. Significance was established at $p<0.05$.

\section{Results}

The reference population included 1776 patients who had osteoarthritis and received HA. Of these, 415 received TJA between 2002 and 2008; 189 patients had not received HA and 226 had received HA prior to TJA. Two hundred and twenty-eight patients who had TJA were excluded for having previous intra-articular corticosteroid, 34 had previous rheumatoid arthritis, and 28 had previous orthopedic 
surgeries or were excluded because of insufficient data regarding the absence of exclusionary criteria or postoperative follow-up. Demographic characteristics of the study groups and those excluded are given in Table 1. There were no significant differences among demographic characteristics or comorbidity (Table 1), or between groups on physical examination of the knee pre- and postoperative one-year follow-up (Table 2). There was a small difference in WOMAC presurgery in the HA versus no-HA groups but this was not significantly different (Table 2). The time from the last HA injection to TJA was $21 \pm 5$ months (range 7-26 months). There were no significant differences in noninfection adverse events. Superficial and deep infections postTJA were not significantly different between groups (Table 2). In particular, there were 18 infected TJAs in the HA versus 21 in the no-HA groups. Seventy-eight patients received high molecular weight HA. When the type of HA was considered; that is, higher molecular weight ( $>1.2$ million $\mathrm{kDa}$ ) versus low molecular weight $(<1.2$ million $\mathrm{kDa})$, only three infections were observed in the low molecular weight group $(p<0.05)$. The demographic, physical examination, WOMAC, and time from HA to TJA were not different for this subset of both groups. Furthermore, total numbers of HA injections prior to TJA had no impact on adverse event rates post-TJA. Five (1 HA, 4 noHA) that became infected required surgical revision. There were non-infection adverse events reported in 28 cases (16 cases HA, 12 cases no-HA). The HA group had seven stiff knees (values of $<90$ degrees), five with severe pain, and two postoperative hemarthrosis. The no-HA group reported one DVT, one stiff knee, one hemarthrosis, and two with severe pain. Six stiff knees required manipulation under anesthesia (5 HA vs. 1 no-HA).

\section{Discussion}

Efficacy of intra-articular HA has been proven to be not only safe but effective. ${ }^{20}$ Intraarticular injections are associated with a low incidence of adverse events, including infection. Adverse events are reported to be greater in higher molecular weight HA products..$^{10,11}$ Definitive treatment of severe knee osteoarthritis that has exhausted conventional interventions is TJA. To our knowledge, there is no evidence to date regarding the risk of infection post-TJA in patients who had previously received intra-articular HA. We observed no difference in the rates of infection (mainly superficial) post-TJA in patients who had or had not received previous HA injections. Further, there was no difference among patients who had received a single versus mul-

Table 1. Baseline characteristics among groups.

\begin{tabular}{lcc} 
Baseline & HA & No HA \\
$\mathrm{N}$ & 226 & 189 \\
Age (years) & $68.3(49-83)$ & $66.1(44-83)$ \\
\hline BMI & $29.8(19.7-41.3)$ & $31.4(21.7-42.5)$ \\
Female & 64 & 61 \\
\hline Follow-up (months) & $3.4(2.7)$ & $3.8(3.3)$ \\
Time from HA (months) & $19(4-31)$ & 1.5 \\
\hline Charlson Index & 1.7 &
\end{tabular}

Table 2. Clinical variables pre- and post-TJA.

\begin{tabular}{lcc} 
Preoperative & HA & No HA \\
Range of motion: extension (degrees) & $5.6(5.7)$ & $5.3(6.4)$ \\
Range of motion: flexion (degrees) & $113.3(11.6)$ & $110.1(13.6)$ \\
\hline WOMAC & $26.4(16.3)$ & $23.2(11.9)$ \\
One-year follow-up & HA & No HA \\
Range of motion: extension (degrees) & $0.8(2.1)$ & $1.6(1.5)$ \\
Range of motion: flexion (degrees) & $113.7(12.8)$ & $117.0(13.5)$ \\
WOMAC & $10.4(13.4)$ & $8.5(12.3)$ \\
Superficial infection (skin) & 14 & 16 \\
\hline Deep infection (articular) & 4 & 5 \\
Adverse events & 18 & 23 \\
\hline
\end{tabular}

tiple courses of HA therapy prior to TJA. Interestingly, we did observe a significant difference in a number of patients who had infection within the HA injection group. Specifically, patients who had received a higher molecular weight HA product had a higher number of post-TJA infections versus those who had received a lower molecular weight treatment. To our knowledge, this is the first report of post-TJA infection rates in patients who had received HA therapy.

Reasons for more infections among those previously exposed to higher molecular weight HA therapy are beyond the scope of this paper. However, a possible mediator could be the higher number of small HA fragments that are precipitated with higher versus lower molecular weight HA treatment. Specifically, HA is broken down into smaller (i.e. $<100 \times 10^{6} \mathrm{Da}$ ) HA fragments of lower molecular weight. ${ }^{21}$ It would be anticipated that higher molecular weight products would produce more smaller HA fragments compared to lower molecular weight HA products. Within some tissues these small molecular weight HA fragments have been reported to activate immunocompetent cells. ${ }^{22}$ For example, in skin, low molecular weight HA fragments induce activation of keratinocytes, which produce $\beta$-defensin- 2 mediated by TLR- 2 and TLR-4 activation and involving a C-FOSmediated protein kinase C-dependent signaling pathway. This activation is not accompanied by an inflammatory response and, hence, $\beta$-defensin may induce a self-defense mecha- nism from an infection by micro-organisms similarly to what could occur within the intraarticular space. Certainly more investigation will be needed to explore the findings of our study in terms of differences between higher and lower molecular weight products.

This is a retrospective analysis of a primary care database. The methods utilized may not be similar to those used in all primary care settings involved in providing intra-articular HA treatment. While the population may be representative of the Southwestern Ontario region, generalization beyond this should be considered carefully. Certainly, different practices with intra-articular HA administration and TJA should be considered. However, this is a very large and robust database with inclusion of multiple comorbidities that tracks patients' progress over several years. Specifically, we included patients who had received intra-articular HA several years prior to their TJA.

In summary, prior intra-articular HA did not show an increased rate of infection post-TJA. A small but significantly greater number of patients who received higher molecular weight HA had infection after TJA compared to those who received lower molecular weight HA. Hence, further investigation of this finding is needed. Different sources of HA, methods of sterilization, and manufacturing should be compared among those receiving TJA. Lastly, further validation of our findings should be conducted in other populations apart from TJA patient groups. 


\section{References}

1. Bellamy N, Campbell J, Robinson V, et al. Viscosupplementation for the treatment of osteoarthritis of the knee. Cochrane Database Syst Rev 2005;2:CD005321.

2. Lo GH, LaValley M, McAlindon T, et al. Intraarticular hyaluronic acid in treatment of knee osteoarthritis: a meta-analysis. JAMA 2003;290:3115-21.

3. Bunyaratavej N, Chan KM, Subramanian $\mathrm{N}$. Treatment of painful osteoarthritis of the knee with hyaluronic acid. Results of a multicenter Asian study. J Med Assoc Thai 2001;84:S576-81.

4. Petrella RJ, Petrella M. A prospective, randomized, double-blind, placebo controlled study to evaluate the efficacy of intraarticular hyaluronic acid for osteoarthritis of the knee. J Rheumatol 2006;33:951-6.

5. Guarda-Nardini L, Tito R, Staffieri A, et al. Treatment of patients with arthrosis of the temporomandibular joint by infiltration of sodium hyaluronate: a preliminary study. Eur Arch Otorhinolaryngol 2002;259:27984.

6. Salk RS, Chang TJ, D'Costa WF, et al. Sodium hyaluronate in the treatment of osteoarthritis of the ankle: a controlled, randomized, double-blind pilot study. J Bone Joint Surg Am 2006; 88:295-302.

7. Blaine T, Moskowitz R, Udell J, et al. Treatment of persistent shoulder pain with sodium hyaluronate: a randomized, controlled trial. A multicenter study. J Bone Joint Surg Am 2008;90:970-9.

8. van den Bekerom MP, Lamme B, Sermon
A, et al. What is the evidence for viscosupplementation in the treatment of patients with hip osteoarthritis? Systematic review of the literature. Arch Orthop Trauma Surg 2008;128:815-23.

9. Neustadt DH. Long-term efficacy and safety of intra-articular sodium hyaluronate (Hyalgan) in patients with osteoarthritis of the knee. Clin Exp Rheumatol 2003;21: 307-11.

10. Petrella RJ. Hyaluronic acid for the treatment of knee osteoarthritis: long-term outcomes from a naturalistic primary care experience. Am J Phys Med Rehabil 2005; 84:278-83.

11. Adams ME, Lussier AJ, Peyron JG. A riskbenefit assessment of injections of hyaluronan and its derivatives in the treatment of osteoarthritis of the knee. Drug Saf 2000;23:115-30.

12. Hammesfahr JF, Knopf AB, Stitik T. Safety of intra-articular hyaluronates for pain associated with osteoarthritis of the knee. Am J Orthop 2003;32:277-83.

13. Goldberg VM, Coutts RD. Pseudoseptic reactions to hylan viscosupplementation: diagnosis and treatment. Clin Orthop Relat Res 2004;419:130-7.

14. Charalambous CP, Tryfonidis M, Sadiq S, et al. Septic arthritis following intra-articular steroid injection of the knee: a survey of current practice regarding antiseptic technique used during intra-articular steroid injection of the knee. Clin Rheumatol 2003;22:386-90.

15. Jamsen E, Varonen U, Huhtala $\mathrm{H}$, et al. Incidence of prosthetic joint infection after primary knee arthroplasty. J
Arthroplasty 2008; dec 3 (Epub ahead of print).

16. Petrella RJ, Merikle E. A retrospective analysis of the prevalence and treatment of hypertension and dyslipidemia in Southwestern Ontario, Canada. Clin Ther 2008;30:1145-54.

17. Charlson ME, Pompei P, Ales KL, et al. A new method of classifying prognostic comorbidity in longitudinal studies: development and validation. J Chron Dis 1987; 40:373-83.

18. Bellamy N, Kirwan J, Boers M, et al. [Recommendations for a core set of outcome measures for future phase III clinical trials in knee, hip, and hand osteoarthritis. Consensus development at OMERACT III]. J Rheumatol 1997;24:799-802.

19. Petrella RJ, Cogliano A, DeCaria J. Combining two hyaluronic acids in osteoarthritis of the knee: a randomized, double-blind, placebo-controlled trial. Clin Rheumatol 2008;27:975-81.

20. Bellamy N, Campbell J, Robinson V, et al. Viscosupplementation for the treatment of osteoarthritis of the knee. The Cochrane Database of Systematic Reviews 2006;2: CD005321, DOI: 10.1002/14651858.

21. Balazs EA. The physical properties of synovial fluid and the specific role of hyaluronic acid. In Disorders of the Knee (Edited by: Helfet AJ). Philadelphia JB Lippincott 1982;61-74.

22. Gariboldi S, Palazzo M, Zanobbio L, et al. Low molecular weight hyaluronic acid increases self-defense of skin epithelium by induction of $\beta$-defensin 2 via TLR2 and TLR4. J Immunol 2008;181:2103-10. 